\title{
Renalase, Catecholamine, and Nitric Oxide Changes before and after Sodium Nitroprusside Administration to Patients Who Develop Post-Coronary Artery Bypass Grafting (CABG) Hypertension
}

\author{
Suna Aydin, $\mathrm{MD}, \mathrm{PhD}^{1}$ \\ ${ }^{1}$ Cardiovascular Surgery Department, Elazig Research and Education Hospital, Health Science University, Elazig, Turkey
}

\section{ABSTRACT}

Background: Hypertension develops at a rate of $33 \%$ to $70 \%$ after coronary artery bypass grafting (CABG) operations. One of the most commonly used drugs to control hypertension is sodium nitroprusside (SNP). Additionally, renalase enzyme destroys catecholamines and mediates the regulation (reduction) of blood pressure. Thus, this clinical study aims to reveal how renalase, catecholamines, and nitric oxide (NO) change and how certain hemodynamic parameters are affected in randomly and prospectively selected individuals who are administered SNP for the treatment of blood pressure elevation within 6 to 8 hours after CABG surgery.

Methods: The study included 26 patients who developed hypertension after CABG, 12 patients who had normal blood pressure after CABG, and 22 healthy individuals. Renalase and catecholamine levels of the patients were measured by using ELISA method and NO levels were determined by spectrophotometry.

Results: Renalase and NO levels of the patients who developed hypertension after CABG were found to be statistically significantly lower than those of healthy controls and patients who did not develop hypertension after CABG, although catecholamine levels were significantly higher in the former. After SNP was started, renalase and NO levels increased, and a significant decrease was observed in the catecholamine levels. Additionally, administration of SNP produced a slight increase in the heart rate and a decrease in systolic arterial pressure (SAP), diastolic arterial pressure (DAP), and mean arterial pressure (MAP).

Conclusion: SNP elevates NO and renalase levels; thus, administration of renalase preparations, which act in the

Received March 12, 2018; accepted May 24, 2018.

This study was orally presented at the 3rd International Conferences on Cardiovascular Medicine and Cardiac Surgery, held on 7uly 5-6, 2018 in Golden Tulip Berlin - Hotel Hamburg, Berlin, Germany.

Correspondence: Suna Aydin MD, PbD, Cardiovascular Surgery Department, Elazig Research and Education Hospital, Health Science University, Elazig 23119, Turkey; +90-5327856168; fax: +90-424-2379138 (e-mail: cerrab52@botmail.com). destruction of catecholamines to contain persistent hypertension that develops in association with catecholamine elevation after CABG surgery, along with SNP and other medications used to lower blood pressure can be an effective therapeutic method to control hypertension.

\section{INTRODUCTION}

Coronary arteries that supply blood to the heart can narrow down or be blocked because of numerous risk factors over time, and this condition is corrected by coronary artery bypass grafting (CABG) operation [Aydin 2013]. One of the most common complications encountered in this surgery is hypertension that develops at about a rate of $33 \%$ to $70 \%$ during the early postoperative period [Whelton 1980]. This hypertensive condition developing in the early postoperative period is attributed primarily to elevated epinephrine and norepinephrine levels [Sealy 1967]. Besides epinephrine and norepinephrine, humoral factors such as increased plasma renin activation (RAA), and autonomous reflexes are involved in the etiology of vasoconstriction [Kobori 2007]. Additionally, temporary hypertension may occur as the depth of anesthesia increases during the operation and when awareness starts to come back after anesthesia [Momota 2010]. After CABG, hypertension may arise in association with increased adrenergic activity [de Champlain 1990], unawareness caused by anesthesia, hypothermia, and painful stimuli [Musizza 2010].

Table 1. Comparison of Mean Hemodynamic Parameters CABG Patients with and without Hypertension Developed after CABG

\begin{tabular}{lcc}
\hline Parameters* & Normal Tension & Hypertension \\
\hline CVP $(\mathrm{mmHg})$ & $7.9 \pm 1.1$ & $8.2 \pm 1.3$ \\
MAP (mmHg) & $87 \pm 7.2$ & $93 \pm 6.8$ \\
MPAP (mmHg) & $23 \pm 2.1$ & $24 \pm 1.9$ \\
HR (time/min) & $86 \pm 2.1$ & $97 \pm 3.4 \dagger$ \\
\hline
\end{tabular}

*CVP, central venous pressure; MAP, mean arterial pressure; MPAP, mean pulmonary arterial pressure; HR, heart rate.

$\dagger P<.05$ compared with and without hypertension developed after CABG. 


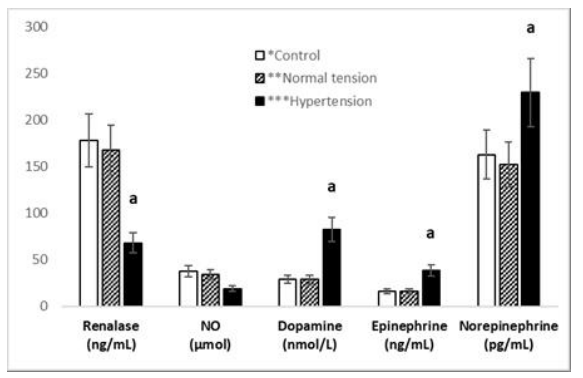

Figure 1. Comparison of renalase, nitric oxide, dopamine, epinephrine, and norepinephrine levels of control and patients after 6-8 hours after coronary artery bypass grafting without any treatment. The difference between the patients who developed hypertension and those who did not and the control cases was statistically significant. *Healthy control. $* *$ Normal tension after CABG. ***Hypertension developed after CABG. $\mathrm{a} P<.05$ compared with and without hypertension developed after CABG.

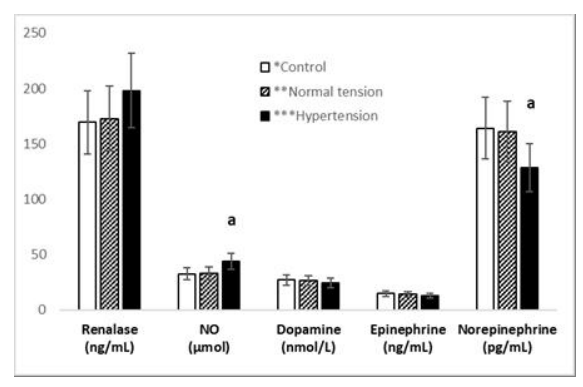

Figure 2. Comparison of renalase, nitric oxide, dopamine, epinephrine, and norepinephrine levels between the group who developed hypertension after coronary bypass surgery (and was administered SNP within 24 hours), the group who did not develop hypertension (and did not receive any administration), and the control group (not subjected to any procedure). *Healthy control. **Normal tension after CABG. ***Hypertension developed after $C A B G$. aP $<.05$ compared with and without hypertension developed after CABG.

Furthermore, hypertension developing after CABG operation was reported to be related to increased systemic vascular resistance $(\mathrm{SVR}=$ mean arterial pressure $[\mathrm{MAP}]-$ central venous pressure $[\mathrm{CVP}] \times 80 /$ cardiac output $[\mathrm{CO}]$ ) in the presence of almost normal CO [Whelton 1980; Lomivorotov 2017].

In addition to all these, hypertension seen in the postCABG operation might be associated with the amounts of renalase secreted by kidneys [Xu 2005]. This is because renalase, with a molecular weight of $38 \mathrm{kD}$ and its gene location on the 10th chromosome (q.23.33), is a flavin adenine dinucleotide (FAD)-dependent monoamine oxidase and metabolizes catecholamines (dopamine, epinephrine, and norepinephrine) [Xu 2005; Hennebry 2010]. Regulating the myocardial contractility, heart rate (HR), and the tonus of resistance vessels, catecholamines play a key role in the regulation of blood pressure [Mannelli 1990]. It was reported that renalase amounts decreased in rats with induced ischemia/ reperfusion injury; and when the animals were administered renalase, circulating catecholamines were reduced, and blood pressure dropped significantly [Lee 2013; Zhao 2015]. Thus,

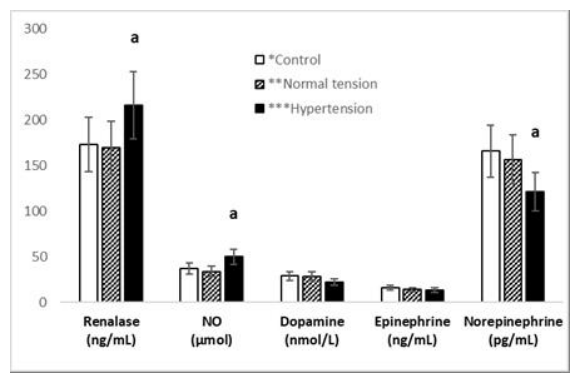

Figure 3. Comparison of renalase, nitric oxide, dopamine, epinephrine, and norepinephrine levels between the group who developed hypertension after coronary bypass surgery (and was administered SNP within 48 hours), the group who did not develop hypertension (and did not receive any administration), and the control group (not subjected to any procedure). $\mathrm{a} P<.05$ compared with and without hypertension developed after CABG.

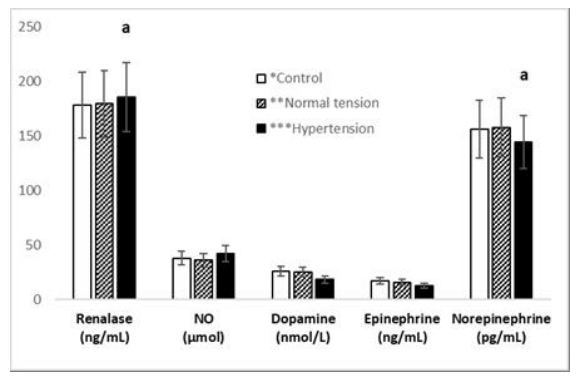

Figure 4. Comparison of renalase, nitric oxide, dopamine, epinephrine and norepinephrine levels between the group who developed hypertension after coronary bypass surgery (and was administered SNP within 72 hours), the group who did not develop hypertension (and did not receive any administration), and the control group (not subjected to any procedure). $a P<.05$ compared with and without hypertension developed after CABG.

renalase is an enzyme of central importance in the regulation of blood pressure [Mannelli 1990; Xu 2005; Hennebry 2010; Lee 2013; Zhao 2015]. Renalase is released to the blood primarily from the kidneys, and it is synthesized, to a lesser extent, in the heart, skeletal muscle, and small intestines [Xu 2005; Hennebry 2010]. It was noted that there was a partial correlation between renalase quantities and hydrogen peroxide production, but a direct connection with superoxide production [Wang 2014]. Hence, all the data suggest that examining the fate of the renalase enzyme may be of key importance in the etiopathophysiology of hypertension following CABG.

A number of agents are used in the treatment of hypertension that develops after (within the first 6 to 8 hours) CABG operation [Leslie 1994; Chobanian 2003; Varon 2008]. One of the most commonly used medications to address this hypertension problem following CABG surgery is sodium nitroprusside (SNP) [Friederich 1995; Gibson 2017]. Acting directly on the vessel smooth muscles, the drug dilates both arterioles and venules and hence lowers blood pressure. When it enters the smooth muscle cell, nitric oxide (NO) is produced, and this is the metabolite responsible for the pharmacological effect of the drug [Gibson 2017]. 
Table 2. Comparison of Certain Demographic and Preoperative Laboratory Variables of the Patients before CABG and Control

\begin{tabular}{lccc}
\hline Parameters* & Control $(\mathrm{n}=22) \dagger$ & Normal Tension $(\mathrm{n}=12) \ddagger$ & Hypertension $(\mathrm{n}=26) \S$ \\
\hline Gender $(\mathrm{n} / \mathrm{n}$ [female/male]) & $15 / 7$ & $72 \pm 13.2$ & $18 / 8$ \\
Group age (years) & $68 \pm 10.2$ & $28.9 \pm 6.8$ & $72 \pm 15.1$ \\
Body mass index $\left(\mathrm{kg} / \mathrm{m}^{2}\right)$ & $26.7 \pm 4.4$ & $128 \pm 7.9$ & $29.1 \pm 6.3$ \\
Systolic blood pressure $(\mathrm{mmHg})$ & $118 \pm 9.4$ & $89 \pm 8.8$ & $135 \pm 9.9$ \\
Diastolic blood pressure $(\mathrm{mmHg})$ & $84 \pm 8.3$ & $102 \pm 8.8$ & $92 \pm 7.9$ \\
Blood glucose (mg/dL) & $94 \pm 7.2$ & $139 \pm 2.9$ & $96 \pm 6.5$ \\
Sodium (mEq/L, mean $\pm \mathrm{SD})$ & $141 \pm 3.2$ & $4.77 \pm 0.3$ & $143 \pm 3.1$ \\
Potassium (mEq/L, mean $\pm \mathrm{SD})$ & $4.68 \pm 0.2$ & $4.5 \pm 1.4$ & $4.62 \pm 0.3$ \\
Uric acid $(\mathrm{mg} / \mathrm{dL}$, mean $\pm \mathrm{SD})$ & $4.2 \pm 1.2$ & $1.1 \pm 0.2$ & $4.7 \pm 1.4$ \\
Creatinine $(\mathrm{mg} / \mathrm{dL})$ & $0.6 \pm 0.1$ & $1.3 \pm 0.2$ \\
\hline
\end{tabular}

*SD, standard deviation.

†Healthy control.

$\ddagger$ Normal tension after CABG.

$\S$ Hypertension developed after CABG.

Based on the foregoing information, the aim of this study is examine and compare the effects of SNP (converted to $\mathrm{NO}$ ), administered to lower the blood pressure in patients who develop hypertension within the first 6 to 8 hours after CABG operation, on the amounts of circulating NO, catecholamines (epinephrine [catabolite, metanephrine], norepinephrine [catabolite, normetanephrine], dopamine [precursor of epinephrine and norepinephrine]), and the amounts of the renalase enzyme which is involved in epinephrine and norepinephrine destruction, as well as its positive and negative effects on hemodynamic parameters.

\section{MATERIALS AND METHODS}

This retrospective/prospective study was started after the approval (meeting number 14, issue no. 05, dated Oct. 26, 2017) of the local ethics committee of the School of Medicine of Firat University and the informed consents of the patients were obtained. It registered 26 coronary artery bypass cases (18 males, 8 females) who developed hypertension in the early postoperative period, 12 coronary artery bypass cases $(9$ males, 3 females) whose blood pressure was normal, and 22 healthy individuals ( 15 males, 7 females) who presented at the hospital for their routine annual checkup and were found to be healthy. Patients who had a myocardial infarction (MI) in the last 6 months or had chronic ventricular arrhythmia, valvular heart disease, or marked renal hepatic dysfunction were not included in the study. Care was taken to make sure that the cases included in the study were similar in terms of age and body mass index. Patients whose MAP was $>90 \mathrm{mmHg}$, systolic arterial pressure (SAP) was $>140 \mathrm{mmHg}$, and HR ranged between 40 and 130 beats per minute despite sufficient analgesia and sedation, and who did not have a conduction abnormality such as second- or third-degree block, did not suffer from hypovolemia or blood loss, did not have marked hepatic failure, did not have ventricular arrhythmia, and had normal fluid and electrolyte balance and pulmonary functions were included in the study [Erturk 1998]. As reported earlier, the main target in this study was to have MAP in the 80 to $90 \mathrm{mmHg}$ range and pulmonary capillary wedge pressure (PCWP) and CVP in the 0 to $5 \mathrm{mmHg}$ ranges [Erturk 1998]. Blood samples of $5 \mathrm{~mL}$ were collected from control group cases once and put into plain biochemistry tubes and tubes containing $3 \mathrm{~mL}$ of heparin. From coronary artery bypass cases, samples of $5 \mathrm{~mL}$ were taken in the postoperative 6-8hour period, as well as at 24th, 48th, and 72nd postoperative hours, and put into plain biochemistry tubes and into tubes containing $3 \mathrm{~mL}$ heparin (green capped). The samples were centrifuged at $4000 \mathrm{rpm}$, and the sera and plasma were stored at $-80 \mathrm{oC}$ until the time of analysis. All patients underwent the CABG operation through standard premedication, anesthesia, and bicaval, aortic cannulation [Lema 2009]. Patients who developed hypertension after coronary artery bypass operation were started on SNP at a dose of $0.5 \pm 0.2(\mu \mathrm{g} / \mathrm{kg}$ per minute) and administered a maintenance infusion dose of $1.0 \pm 0.1(\mu \mathrm{g} / \mathrm{kg}$ per minute) [Erturk 1998]. The maximum SNP dose used was $1.9 \pm 0.3(\mu \mathrm{g} / \mathrm{kg}$ per minute). These doses were determined according to a previous study and routine administration principles. Factors that were considered in discontinuing the treatment were hypotension, normal blood pressure, surgical hemorrhage, insufficient efficiency, and side effects such as arrhythmia [Erturk 1998]. Patients who did not develop hypertension after coronary bypass surgery were not given any medication to control blood pressure, but were monitored only.

\section{Analytical Measurements}

Nitric Oxide (NO) Analysis. To quantify NO (micromol/L), nitrate was reduced to nitrite in the presence of nitrate reductase enzyme, and the absorbance of the 
Table 3. Comparison of Certain Time-Depended Postoperative Laboratory Variables in the CABG Patients Who Did and Did Not Develop Hypertension with and without SNP Treatment

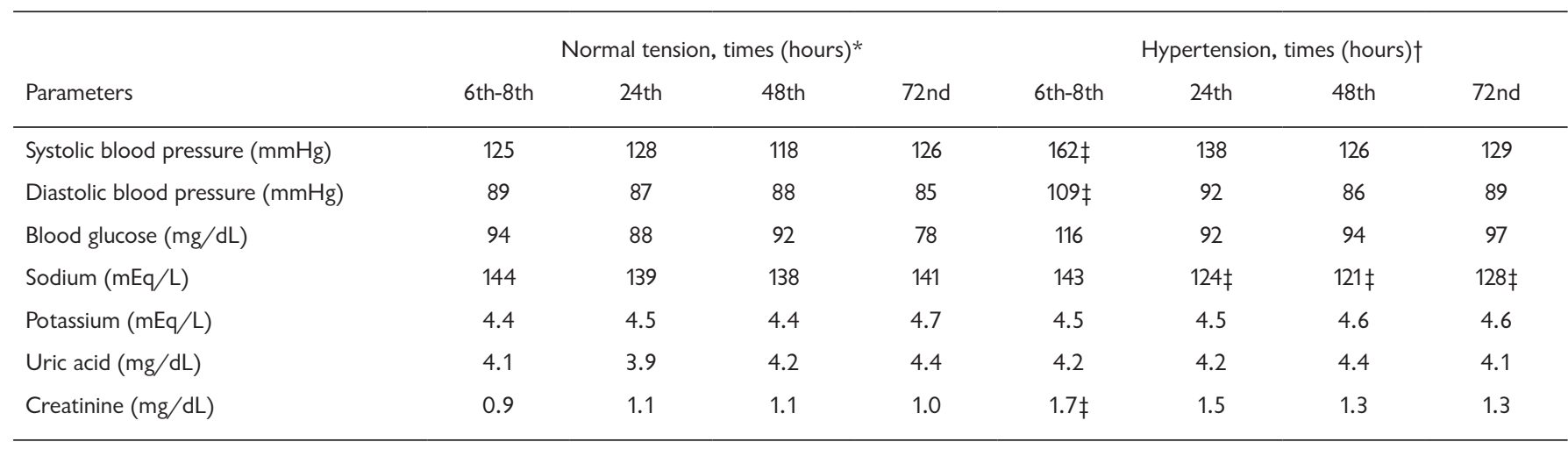

*Normal tension after CABG without treatment SNP.

†Hypertension developed after CABG and treated with SNP

$\ddagger \mathrm{P}<.05$ compared with and without hypertension developed after CABG.

complex formed by the reaction between nitrite and sulfanilamide and $N$-ethylenediamine dihydrochloride was measured at $545 \mathrm{~nm}$ by spectrophotometry [Gayathri 1999; Narayanai 2009]. Hemodynamic and biochemical parameters were obtained from the patients' records. Details about the analyses of hemodynamic parameters can be found in the article published earlier by our team [Aydin 2014].

Catecholamines and Renalase Analysis. Serum renalase (cat. no., 201-12-5371), dopamine (cat. no., 201-12-1302), epinephrine (metanephrine [MN], cat. no., 201-12-0980) were from SunRed Biological Technology Co. Ltd (Shanghai, China). Norepinephrine levels (cat. no., OPH1F2001) from Optima Tibbi ve Kimyevi, Trade Ltd (Istanbul, Turkey) were established by using the ELISA method, as instructed in the producer company's catalogue. It was noted in the company's catalogue that the kit's renalase detection range was 3-700 $\mathrm{ng} / \mathrm{mL}$, intra-assay value was $<10 \%$, and interassay value was $<12 \%$. Also, as stated by the kits' producers, assay range was 8-2000 nmol/L for dopamine, 0.3-60 ng/ $\mathrm{mL}$ for epinephrine $(\mathrm{MN})$, and $18-1000 \mathrm{pg} / \mathrm{mL}$ for norepinephrine. As for intra- and interassay values of the kits as reported by the producers, intra-assay values were $10 \%$ for dopamine, $10 \%$ for norepinephrine, and $12 \%$ for norepinephrine, whereas the interassay values were $12 \%$ for dopamine, $12 \%$ for metanephrine, and $11 \%$ for norepinephrine.

Statistical Analyses. SPSS 21.0 for Windows (IBM, Armonk, NY, USA) software was used in all statistical analyses. Data obtained in the study were expressed as mean \pm standard deviation. Values of $P<.05$ were considered statistically significant. Kruskal-Wallis one-way variance analysis was employed in the comparison of parameters between groups. Dual comparisons between groups were conducted by using Mann-Whitney $U$ test. The correlations between group parameters were examined with Spearman correlation test.

\section{RESULTS}

During the SNP treatment, only 2 patients had hypotension, and there was not any other complication. Treatment of these 2 patients who developed hypotension was interrupted for a short time and resumed after volume replacement. When the patients were compared in terms of number of grafts used $(2.9 \pm 0.4)$, perfusion time (98.6 $\pm 9.3 \mathrm{~min}$.), and aortic cross-clamp duration $(57.6 \pm 4.2 \mathrm{~min}$.), there was not any difference between the patients who did and did not develop hypertension. Hemodynamic parameters of the patients who had and who did not have hypertension after coronary bypass operation are summarized in Table 1. Certain preoperative biochemical values in the coronary bypass patients who did and did not develop hypertension with control values are given in Table 2 . Certain time-dependent postoperative laboratory variables in the coronary bypass patients who did and did not develop hypertension with and without SNP treatment after coronary bypass surgery are presented in Table 3. SNP administration caused a statistically significant drop in sodium (Table 3). Dopamine, epinephrine, and norepinephrine levels in the first 6th to 8th hours were statistically significantly higher in the patients who developed hypertension, in comparison to the levels in the control group, whereas $\mathrm{NO}$ value was slightly, but not statistically significantly higher, and renalase levels were statistically significantly lower (Figure 1). After SNP was started, NO levels at 24th (Figure 2), 48th (Figure 3) and 72nd hours (Figure 4) showed a significant increase relative to the levels in the 6th to 8 th hours (Figure 1), and a similar increase was observed in renalase levels. The increase in renalase levels, however, was not statistically significant at the 24th hour, but was found significant at the 48th and 72nd hours (Figures 2-4). Elevated renalase levels reduced catecholamines (dopamine, metanephrine, and normetanephrine) statistically significantly at the 24th, 48th, and 72nd hours (Figures 2-4). Additionally, there was a positive linear correlation between renalase and 
NO levels in the hypertension patients who received SNP and a negative correlation between catecholamines and these 2 parameters (renalase and $\mathrm{NO}$ ).

\section{DISCUSSION}

Hypertension arising in the early period after CABG operation was first reported in the literature by Estafanous and colleagues in 1973 [Estafanous 1973] and remains a current problem. SNP, a drug that rapidly takes MAP under control, is commonly used in clinical practice for the treatment of hypertension developing in the early post-CABG period [Erturk 1998; Hottinger 2014]. The effect of the drug on left ventricular functions is established by monitoring left ventricular stroke index (LVSWI), PCWP, CO, cardiac index $(\mathrm{CI} ; \mathrm{CI}=\mathrm{CO} /$ body surface area $[\mathrm{BSA}])$, and stroke volume $(\mathrm{SV} ; \mathrm{SV}=\mathrm{CO} \times 1000 / \mathrm{HR})$ [Muralidhar 2002]. The present study examined for the first time whether the renalase enzyme had a role in the etiopathology of hypertension developing in the early post-CABG period. Renalase is an enzyme that metabolizes dopamine, epinephrine, and norepinephrine [Xu 2005]. Previous studies showed a direct relation between hypertension in the early postoperative period and elevated epinephrine and norepinephrine levels [Kobori 2007; Sealy 1967]. In this study, renalase enzyme levels in the first blood samples collected from the patients who developed postoperative hypertension were found statistically significantly lower than those in the healthy controls. There was an inverse proportion between reduced renalase levels and creatine; that is, as creatine levels increased, renalase levels decreased. Patients who developed hypertension in the first 6 to 8 postoperative hours were put on SNP. Blood samples taken 24 hours after SNP administration was started showed higher renalase levels and partially lower creatine levels. These data suggest that CABG operation places a certain amount of burden on the kidneys and partially destroys the cells releasing renalase enzyme, resulting in a decrease in the renalase enzyme, which cannot be released from the cells in sufficient amounts. Then, either because SNP administration improves the renal functions or the kidneys resume their functions over a period of about 16 hours, renalase levels in the samples collected at 24th, 48th and 72nd hours are elevated. Elevated renalase may have mediated the lowering of blood pressure down to the normal range by metabolizing dopamine, epinephrine, and norepinephrine [Sealy 1967]. Stress is the major trigger of epinephrine secretion [Tilders 1985; Ranabir 2011]. Stress reaches the highest levels in all surgical interventions, including the CABG surgery [Najmaii 2006]. In case of danger or physical exertion (in response to provocation), stored catecholamines are freed through exocytosis [Telenius-Berg 1987; Messan 2017]. Hypertension that is seen after CABG surgery may have resulted from the increased release of provoked catecholamines or the statistically significant reduction in the renalase enzyme, as reported here, which destroys catecholamines. It was also found in this study that SNP administration caused a statistically significant rise in the HR. Three clinical studies conducted at the London Chest Hospital, London, and the Maastricht Hospital, the Netherlands
[Undenvood 1989; Lavvrence 1993], and the Cardiovascular Surgery Department of Dokuz Eylül University's School of Medicine reported a marked increase in the HR with SNP administration [Ertuk, 1998].

The present study also showed that patients with hypertension had lower sodium levels. This is thought to be caused by elevated catecholamine, and particularly, dopamine levels. As it is known, the proximal tube produces dopamine, which contributes to the regulation of sodium discharge [Takemoto 1992; Wang 2009]. Once dopamine is released from the proximal tube cells, it cannot reenter tubular cells [Wang 2009]; therefore, after the release, the intracellular enzymes monoamine oxidase (MAO) and catechol-O-methyltransferase (COMT) can no longer regulate dopamine. In consideration of the evidence about renalase and dopamine, it is obvious that renalase mediates the regulation of dopamine-dependent natriuresis and phosphaturia observed in 5/6-nephrectomized $(5 / 6 \mathrm{Nx})$ and sham rats [Desir 2014; Sizova 2013]. Furthermore, a low-salt diet reduces the activity of aromatic L-amino acid decarboxylase (AADC) and inhibits renal dopamine synthesis and discharge; the opposite will take place in highsalt conditions [Burns 1996]. In other words, high sodium increases dopamine synthesis and thus acts as a mediator in increasing the blood pressure [Quelhas-Santos 2013]. That is the reason why salt intake is restricted in patients who have sodium-mediated hypertension.

SNP, which is administered to take postoperative hypertension under control, is NO mediated and lowers the blood pressure by dilating arterioles and venules [Grossi 2005]. Therefore, the present study also investigated the NO changes in the patients who were administered SNP to control postoperative hypertension. Although NO levels in hypertensive patients before administration were lower, NO levels after SNP administration showed a time-dependent increase. This indicates that SNP initiates excessive release of NO from the entire endothelium, and because $\mathrm{NO}$ is a potent vasodilator, it lowers the blood pressure by decreasing SVR [Erturk 1998]. SNP, administered to take postoperative hypertension under control, and epinephrine affect the cardiovascular system and bring about an increase in the blood flow of striated muscles. Epinephrine also supplies the needed energy to the body in times of stress by inducing lipolysis in the adipose tissue, glycogenolysis in the muscles, and glycogenolysis and gluconeogenesis in the liver [Dufour 2009]. Hence, it is suggested that SNP, epinephrine, and renalase work closely to take high blood pressure under control.

In conclusion, although the low number of cases can be considered a limitation of the study, the data suggest that renalase measurement in cases with high blood pressure resulting from elevated catecholamines after cardiopulmonary bypass might give an idea about catecholamine amounts. As circulating renalase levels increase, blood pressure is lowered through reduced catecholamine levels. Also, the data reported for the first time in this study indicate that renalase preparations can be used in addition to SNP and other blood pressure-lowering (or the opposite in case of hypotension as recently reported [Aydin, 2018]) drugs to manage persistent postoperative hypertension in the future. 
It was reported in a previous study that renalase administration to cases reduced plasma epinephrine levels by $82 \%$, L-DOPA levels by $63 \%$, and dopamine levels by $31 \%$ and thus lowered blood pressure.

\section{ACKNOWLEDGMENTS}

I also would like to extend my heartfelt thanks to my cardiac surgery advisor Prof. Dr. Mehmet Nesimi Eren (Dicle University), to whom I will feel indebted forever for his valuable contributions to my education, and to Prof. Dr. Suleyman Aydin at the Medical Biochemistry Department of School of Medine of Firat University, for his kind help in biochemical analyses.

\section{REFERENCES}

Aydin S, Aydin S, Eren MN, et al. 2013. The cardiovascular system and the biochemistry of grafts used in heart surgery. Springerplus 2:612.

Aydin S, Eren MN. 2018. Renalase decisive enzyme about the postoperative term hypotension after coronary artery bypasses graft surgery. Blood Heart Circ, 2:1-4.

Aydin S, Kalayci M, Eren MN, Yilmaz M, Aydin S. 2014. Analysis of hemodynamics and biochemical profile during coronary artery bypass surgery using cardiopulmonary bypass. Heart Health Open Access 1:1.

Burns N, Brett L, Olverman HJ, Nagatsu T, Lee MR, Williams BC. 1996. The role of L-aromatic amino acid decarboxylase in serotonin-stimulated aldosterone secretion in response to salt intake. Endocr Res 22:577-8.

Chobanian AV, Bakris GL, Black HR, et al. 2003. Seventh report of the Joint National Committee on Prevention, Detection, Evaluation, and Treatment of High Blood Pressure. Hypertension. 42:1206-52.

de Champlain J. 1990. Pre- and postsynaptic adrenergic dysfunctions in hypertension. J Hypertens Suppl 8:S77-85.

Desir GV, Peixoto AJ. 2014. Renalase in hypertension and kidney disease. Nephrol Dial Transplant 29:22-8.

Dufour S, Lebon V, Shulman GI, Petersen KF. 2009. Regulation of net hepatic glycogenolysis and gluconeogenesis by epinephrine in humans. Am J Physiol Endocrinol Metab 297:E231-5.

Erturk M, Acikel U, Silistreli E, Hazan E, Oto O. 1998. The comparison of the efficacies of isradipine and sodium nitroprusside on the hypertension after coronary bypass operations. Turk J Thorac and Cardiovasc Surg 6:190-7.

Estafanous FG, Tarazi RC, Viljoen JF, el-Tawil MY. 1973. Systemic hypertension following myocardial revascularization. Am Heart J 85:732-8.

Friederich JA, Butterworth JF. 1995. Sodium nitroprusside: twenty years and counting. Anesth Analg 81:152-62.

Gayathri N, Balasubramanian N. 1999. Spectrophotometric determination of nitrogen dioxide, nitrite and nitrate with Neutral Red. Analusis 27:174-81.

Gibson CM, Davis S, Bradford D. 2017. Examining the use of sodium nitroprusside in coronary artery bypass grafting: is the benefit worth the cost? Hosp Pharm 52:502-7.

Grossi L, D'Angelo S. 2005. Sodium nitroprusside: mechanism of NO release mediated by sulfhydryl-containing molecules. J Med Chem 48:2622-6.
Hennebry SC, Eikelis N, Socratous F, Desir G, Lambert G, Schlaich M. 2010. Renalase, a novel soluble FAD-dependent protein, is synthesized in the brain and peripheral nerves. Mol Psychiatry 15:234-6.

Hottinger DG, Beebe DS, Kozhimannil T, Prielipp RC, Belani KG. 2014. Sodium nitroprusside in 2014: a clinical concepts review. J Anaesthesiol Clin Pharmacol 30:462-71.

Kobori H, Nangaku M, Navar LG, Nishiyama A. 2007. The intrarenal renin-angiotensin system: from physiology to the pathobiology of hypertension and kidney disease. Pharmacol Rev 59:251-87.

Lavvrence CJ, Lestrade A, Chan E, Du Lange S. 1993. Comparative study of isradipine and sodium nitroprusside in the control of hypertension in patients following coronary artery-bypass surgery. Acta Anaesthesiol Scand Suppl 99:48-52.

Lee HT, Kim JY, Kim M, et al. 2013. Renalase protects against ischemic AKI. J Am Soc Nephrol 24:445-55.

Lema G, Urzua J, Jalil R, et al. 2009. Decreased nitric oxide products in the urine of patients undergoing cardiac surgery. J Cardiothorac Vasc Anesth 23:188-94.

Leslie J, Brister N, Levy JH, et al. 1994. Treatment of postoperative hypertension after coronary artery bypass surgery. Double-blind comparison of intravenous isradipine and sodium nitroprusside. Circulation 90(5 Pt 2):II256-61.

Lomivorotov VV, Efremov SM, Kirov MY, Fominskiy EV, Karaskov AM. 2017. Low-cardiac-output syndrome after cardiac surgery. J Cardiothorac Vasc Anesth 31:291-308.

Mannelli M, Pupilli C, Lanzillotti R, Ianni L, Serio M. 1990. Catecholamines and blood pressure regulation. Horm Res 34:156-60.

Messan F, Tito A, Gouthon P, et al. 2017. Comparison of catecholamine values before and after exercise-induced bronchospasm in professional cyclists. Tanaffos 16:136-43.

Momota Y, Kaneda K, Arishiro K, Kishimoto N, Kanou S, Kotani J. 2010. Changes in blood pressure during induction of anesthesia and oral and maxillofacial surgery by type and timing of discontinuation of antihypertensive drugs. Anesth Prog 57:13-7.

Muralidhar K. 2002. Central venous pressure and pulmonary capillary wedge pressure monitoring. Indian J Anaesth 46:298-303.

Musizza B, Ribaric S. 2010. Monitoring the depth of anaesthesia. Sensors (Basel) 10:10896-935.

Najmaii S, Redford D, Larson DF. 2006. Hyperglycemia as an effect of cardiopulmonary bypass: intra-operative glucose management. J Extra Corpor Technol 38:168-73.

Narayanai B, Sunil K. 2009. A spectrophotometric method for the determination of nitrite and nitrate. Eurasian J Anal Chem 4:204-14.

Quelhas-Santos J, Sampaio-Maia B, Simões-Silva L, et al. 2013. Sodiumdependent modulation of systemic and urinary renalase expression and activity in the rat remnant kidney. J Hypertens 31:543-52.

Ranabir S, Reetu K. 2011. Stress and hormones. Indian J Endocrinol Metab 15:18-22.

Sealy WC. 1967. Coarctation of the aorta and hypertension. Ann Thorac Surg 3:15-28.

Sizova D, Velazquez H, Sampaio-Maia B, Quelhas-Santos J, Pestana M, Desir GV. 2013. Renalase regulates renal dopamine and phosphate metabolism. Am J Physiol Renal Physiol 305:F839-44. 
Takemoto F, Cohen HT, Satoh T, Katz AI. 1992. Dopamine inhibits $\mathrm{Na} / \mathrm{K}-\mathrm{ATP}$ ase in single tubules and cultured cells from distal nephron. Pflugers Arch 421:302-6.

Telenius-Berg M, Adolfsson L, Berg B, et al. 1987. Catecholamine release after physical exercise. A new provocative test for early diagnosis of pheochromocytoma in multiple endocrine neoplasia type 2. Acta Med Scand 222:351-9.

Tilders FJ, Berkenbosch F, Vermes I, Linton EA, Smelik PG. 1985. Role of epinephrine and vasopressin in the control of the pituitary-adrenal response to stress. Fed Proc 44(1 Pt 2):155-60.

Varon J, Marik PE. 2008. Perioperative hypertension management. Vasc Health Risk Manag 4:615-27.

Wang L, Velazquez H, Moeckel G, et al. 2014. Renalase prevents AKI independent of amine oxidase activity. J Am Soc Nephrol 25:1226-35.

Wang X, Armando I, Upadhyay K, Pascua A, Jose PA. 2009. The regulation of proximal tubular salt transport in hypertension: an update. Curr Opin Nephrol Hypertens 18:412-20.

Whelton PK, Flaherty JT, MacAllister NP, et al. 1980. Hypertension following coronary artery bypass surgery. Role of preoperative propranolol therapy. Hypertension 2:291-8.

Xu J, Li G, Wang P, et al. 2005. Renalase is a novel, soluble monoamine oxidase that regulates cardiac function and blood pressure. J Clin Invest 115:1275-80.

Zhao B, Zhao Q, Li J, Xing T, Wang F, Wang N. 2015. Renalase protects against contrast-induced nephropathy in Sprague-Dawley rats. PLoS One 10:e0116583. 